\title{
Determination of density contrasts for a three-dimensional sub-surface intermediate layer
}

\author{
I. L. Ateya ${ }^{1}$ and S. Takemoto ${ }^{2}$ \\ ${ }^{1}$ Department of Geomatics Engineering and Geo-spatial Information Systems, Jomo Kenyatta University of Agriculture and Technology, \\ P. O. Box 62000, Nairobi, Kenya \\ ${ }^{2}$ Department of Geophysics, Kyoto University, Kitashirakawa Oiwake-cho, Sakyo-ku, Kyoto 606-8502, Japan
}

(Received January 9, 2003; Revised August 29, 2003; Accepted August 29, 2003)

\begin{abstract}
The quantitative determination of the variable density contrasts for an intermediate horizontal layer has been demonstrated. In particular a sub-surface dipping dike with a priori depth-dependent density contrasts was adopted as a forward model to project gravity anomaly effect above the Earth surface. The sub-surface location and density contrasts in a series of intermediate horizontal layers in the causative dipping dike structure have been recovered by means of inversion analysis. Density contrasts recovery errors of less than 8.0 percent were realized to a depth of $2.00 \mathrm{~km}$ on a maximum synthetic gravity anomaly effect of $10.0 \mathrm{mGals}$ that is better in comparison to constant density models. Finally to demonstrate the efficacy of the inverse analysis in the study, the entire process was successfully applied to real field data, i.e., residual gravity anomaly for a micro-gravimetry site and/or localized structures in Matsumoto Basin, Chubu District-Japan.
\end{abstract}

Key words: Density contrasts, intermediate horizontal layer, sub-surface dipping dike.

\section{Introduction}

The solution of problems in inverse theory and/or downward continuation of potential fields into arbitrary regions of the lower half-space are extensive in geophysical literature. Notably the research works of Savinsky (1963, 1967, 1984, 1995) and Savinsky et al. (1981) among other authors. For example, Savinsky et al. (1981) determines by downward continuation in the lower half-space the disturbing masses in a horizontal layer. Further, the entire lower half-space is similarly considered for disturbing masses for an intermediate layer from observed potential fields, i.e., gravity and magnetism in Savinsky (1984).

When density varies in the lower half-space or within a sub-surface structure it results in what is referred to as variable density. The density contrasts are then the difference between the structure's density and density of surrounding geological materials assumed to be homogeneous. In seeking to explain the gravity anomalies, density variations are considered and these have a direct relationship with the subsurface structure's disturbing masses. In order to distinguish the different materials, then density contrasts are sought in the different layers in the lower half-space.

The gravity anomaly of a complicated two-dimensional source having arbitrary surfaces and the density distribution separated by either horizontal or vertical direction can be calculated using a combination of a closed form solution or numerical interpretations (Ruotoistenmaki, 1992). Rao (1986a, 1990) considered the problem of variable density contrasts and derived the gravity anomalies of prisms and trapezoids having second-degree polynomial density distributions in the

Copy right(C) The Society of Geomagnetism and Earth, Planetary and Space Sciences (SGEPSS); The Seismological Society of Japan; The Volcanological Society of Japan; The Geodetic Society of Japan; The Japanese Society for Planetary Sciences. vertical directions.

Modeling of regular sub-surface structures e.g., dikes and faults, to depict their anomaly patterns in the lower halfspace has been tackled extensively in Geldart et al. (1966) and Telford et al. (1990). Other authors have studied twodimensional gravity models with variable density contrasts, e.g., Cordell (1973), Murthy and Rao (1979), Martin-Atienza and Garcia-Abdeslem (1988). A more general approach is by Guspi (1990) who considered gravity sources bounded by polygons and having polynomial density distribution varying with depth.

$\mathrm{Li}$ and Oldenburg $(1996,1998)$ on the other hand, proposed a sub-surface model of a dipping dike with a constant density throughout its volume. The gravity anomaly effect was then transformed to pseudo-magnetic anomaly and adopted for the recovery of the locations and/or parameters of the sub-surface structures. The sub-surface locations with respect to depth and the lateral variations were a key interest in Li and Oldenburg $(1996,1998)$ contributions.

Assuming a uniform or variable contrasts in the subsurface it is possible to calculate from the potential field signals the probable geometry of the causative structure. Boulanger and Chouteau (2001) adopted a dipping dike model similar to that of $\mathrm{Li}$ and Oldenburg $(1996,1998)$ but introduced the rectangular parameterizations, i.e., density units to the sub-surface structure.

In our case, we adopt a variant of the dipping dike model and introduce a depth-dependent density contrasts factor in the sub-surface structure. Effectively the density contrasts in the structure with respect to depth of horizontal layers are known a priori. We attempt the recovery of the sub-surface density contrasts in the dipping dike at varying depths and intermediate layer heights in three-dimensions. 


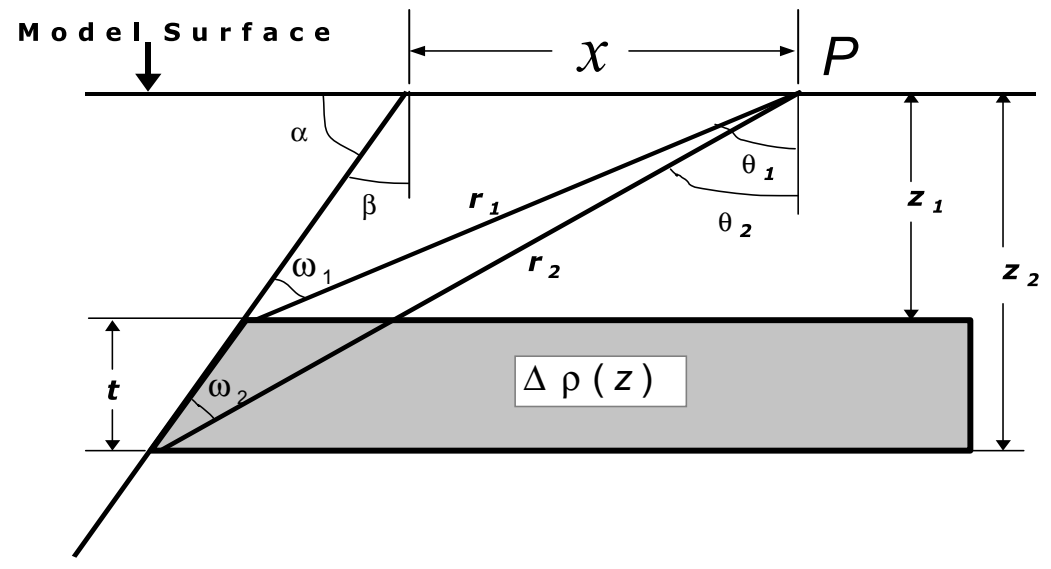

Fig. 1. Parameters and/or variables for gravity anomaly effect of a horizontal slab.

Table 1. Density in the intermediate layers in the dipping dike.

\begin{tabular}{cccc}
\hline Layer & $\begin{array}{c}\text { Depth of layer } \\
(\mathrm{km})\end{array}$ & $\begin{array}{c}\text { Density contrasts } \\
\left(\mathrm{g} / \mathrm{cm}^{3}\right)\end{array}$ & $\begin{array}{c}\text { Differences in } \\
\text { contrasts }\left(\mathrm{g} / \mathrm{cm}^{3}\right)\end{array}$ \\
\hline 1 & 0.000 & -0.525 & 0.000 \\
2 & 0.400 & -0.493 & 0.032 \\
3 & 0.800 & -0.451 & 0.042 \\
4 & 1.200 & -0.409 & 0.042 \\
5 & 1.600 & -0.368 & 0.041 \\
6 & 2.000 & -0.329 & 0.039 \\
7 & 2.400 & -0.290 & 0.039 \\
8 & 2.800 & -0.252 & 0.038 \\
\hline
\end{tabular}

\section{Dipping Dike Model}

Modeling is one of the most important tools in geophysical sciences as it allows a quantitative prediction and establishment of relations to measurements of real objects. In order to understand how a model influences particular potential field data one must be able to calculate theoretical or synthetic data for an assumed contrived Earth model, which constitutes the forward problem. This sometimes involves deriving a mathematical relationship between the data and the contrived model.

\subsection{Location characteristics}

Starting from synthetic gravity anomaly effects, which explain density variations in the sub-surface structure, the goal is to recover the structure density contrasts per each horizontal layer by invoking a valid model. Assuming a sub-surface structure that consists of nearly homogenous sediments we simulate a regular dipping dike-like structure. The causative structure is such that one face, i.e., namely XZ has dip angle of $45^{\circ}$, a second $\mathrm{YZ}$ face resembles a finite horizontal slab and a top XY face is a regular square. The three faces form a regular three-dimensional dipping dike model (cf. Boulanger and Chouteau (2001)

\subsection{Geological materials and density contrasts}

Rock densities prominently feature in the analysis of subsurface structures and therefore in the interpretation of gravity anomalies, it is necessary to estimate the densities of the sub-surface rocks before one can postulate their structure. For this reason it is desirable to adopt some geological data on the densities of the representative rocks in regions where the gravity surveys are made. It should be pointed out that it is not the absolute densities but the density contrasts that are significant. In this study we adopted the geological materials similar to those in Ateya and Takemoto (2002a,b).

The variation in density contrasts with depth can be approximated by a smooth function either quadratic or exponential by least squares fitting of the function to the observed data (Rao, 1986a; Zhang et al., 2001). Further Zhang et al. (2001) studied gravity anomalies of two-dimensional bodies with layers of variable density contrast like rectangular cylinders and inclined fault models. Following after Rao (1986b) and Zhang et al. (2001) we approximated the depthdependent variable density contrasts for a sub-surface dipping dike based on geological materials in Ateya and Takemoto $(2002 a, b)$ as:

$$
\Delta \rho(z)=-0.515+0.109 z-0.003 z^{2}
$$

Table 1 shows the layer density contrasts and the contrasts differences between the respective intermediate horizontal layers. The density contrast at top surface (depth equal zero) is same as the difference between the average alluvial deposits of density $2.12 \mathrm{~g} / \mathrm{cm}^{3}$ and the average reduction density of $2.645 \mathrm{~g} / \mathrm{cm}^{3}$ in the region after Yamamoto et al. (1982), i.e., $\Delta \rho(0)=-0.525$.

Most of the geological materials used in the synthetic modeling are from Central Ranges in the Japan Alps and therefore the reduction density adopted was $2.645 \mathrm{~g} / \mathrm{cm}^{3}$, a value closer the average value of $2.64 \mathrm{~g} / \mathrm{cm}^{3}$ by Yamamoto et al. (1982).

The value was obtained by a least squares method that incorporates the topography that covers an extensive 40,000 $\mathrm{km}^{2}$ with elevation heights ranging from 0 to $3000 \mathrm{~m}$ for which the Earth's sphericity cannot be ignored (Yamamoto et al., 1982).

Table 1 gives the maximum possible values for density contrasts in the horizontal layers assuming homogenous geological materials. The density contrasts in the third column are the dipping dike model values known a priori which we seek to recover by inversion analysis per each horizontal layer. 


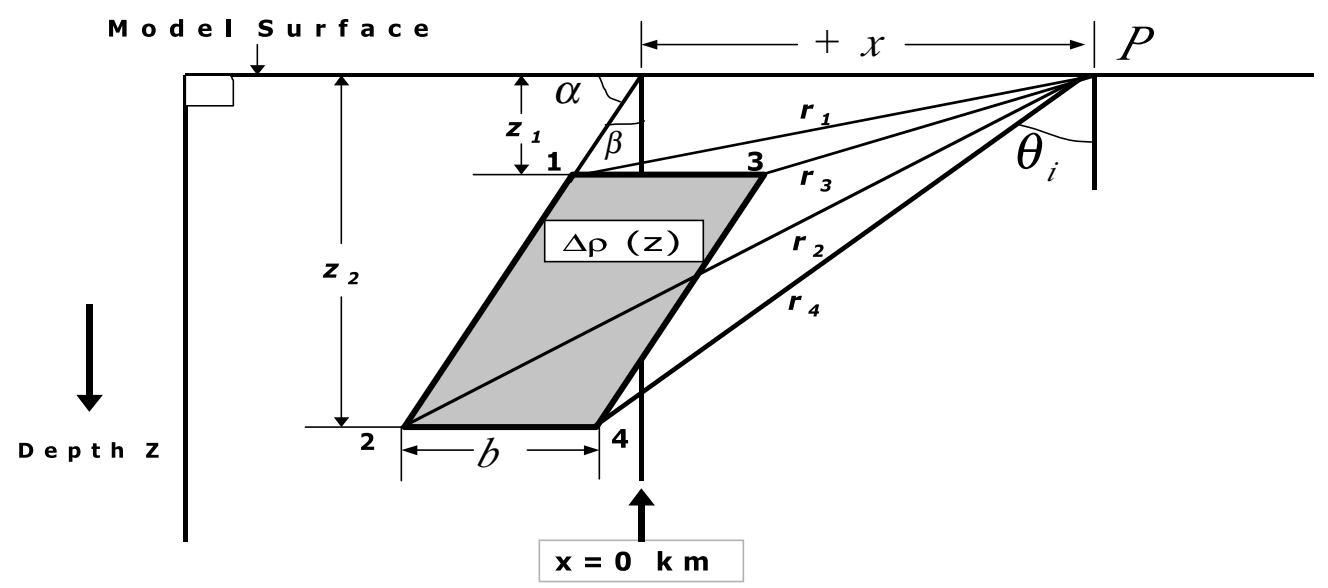

Fig. 2. Parameters and/or variables describing the gravity anomaly effect of a sub-surface dike-like structure.

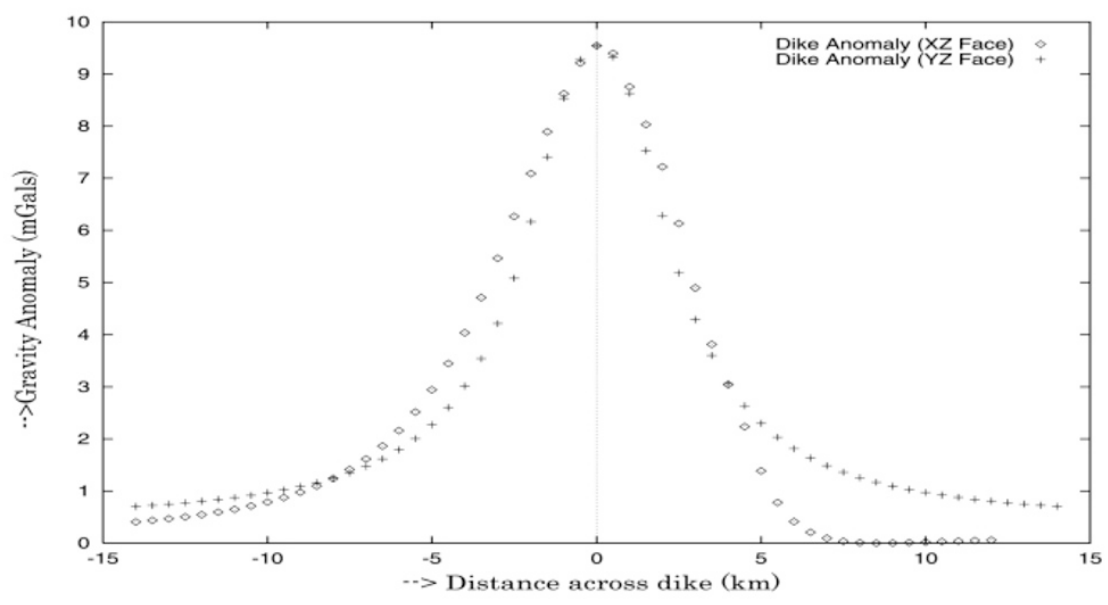

Fig. 3. Gravity anomaly effects of the independent $X Z$ and $Y Z$ dike faces with point-to-point spacing $0.50 \mathrm{~km}$.

\subsection{Gravity anomaly}

For simplicity we assume synthetic data interpolated onto a regular rectangular grid over the sub-surface dipping dike. The top surface of model is assumed to coincide with a flat Earth surface. Independently the $Y Z$ face is modeled as finite sub-surface horizontal slab with the different parameters are shown in Fig. 1 where $x$ is the distance of an observation point $P, t$ is the thickness of slab, $\beta$ is the complement of the dip angle $\alpha$, while $z_{1}$ and $z_{2}$ are the depths to the top and bottom of the slab respectively.

Further, in Eq. (2) $G$ is the gravitational constant while $\Delta \rho(z)$ are variable density contrasts. The depth to top surface, i.e., model Earth surface $z_{1}$ is $0.025 \mathrm{~km}$, depth to the bottom $z_{2}$ is $4.050 \mathrm{~km}$ and widths of $3.050 \mathrm{~km}$ in the regu$\operatorname{lar} x$ and $y$ directions. The gravity anomaly effect $g_{\text {obs }}$, of a two-dimensional finite horizontal slab is given by Geldart et al. (1966) and Telford et al. (1990) as in Eq. (2).

$$
\begin{aligned}
g_{\text {obs }}=2 G \Delta \rho(z)\{ & \left(\frac{\pi t}{2}\right)+\left(z_{2} \theta_{2}-z_{1} \theta_{1}\right)+x\left(\theta_{2}-\theta_{1}\right) \\
& \left.\cdot \sin \beta \cos \beta+x \cos ^{2} \beta \ln \left(\frac{r_{2}}{r_{1}}\right)\right\}
\end{aligned}
$$

The $X Z$ face can be modeled as a sub-surface dike-like structure with its different parameters depicted as in Fig. 2. The distance $x$ is positive when the point $P$ is to the right of central position with all angles being measured in the clockwise direction. The angles $\beta$ and $\theta_{i}$ are measured from the vertical and $\alpha$ from the plane with $z_{i}$ as the depth in the sub-surface. A two-dimensional dike can be obtained by the subtraction of two slabs one being displaced horizontally with respect to the other. The gravity anomaly effect $g_{\text {obs }}$ of such a dike-like structure is given by Geldart et al. (1966) and Telford et al. (1990) as Eq. (3):

$$
\begin{aligned}
g_{\text {obs }}=2 G \Delta \rho(z)[ & z_{2}\left(\theta_{2}-\theta_{4}\right)-z_{1}\left(\theta_{1}-\theta_{3}\right)+\sin \beta \cos \beta \\
& \cdot\left\{x\left(\theta_{2}+\theta_{3}-\theta_{4}-\theta_{1}\right)+b\left(\theta_{4}-\theta_{3}\right)\right\} \\
& \left.+\cos ^{2} \beta\left\{x \ln \left(\frac{r_{2} r_{3}}{r_{1} r_{4}}\right)+b \ln \left(\frac{r_{4}}{r_{3}}\right)\right\}\right]
\end{aligned}
$$

A regular grid spacing of $0.50 \mathrm{~km}$ was adopted in the computation of the gravity anomaly effect for both horizontal slab and dike to $\pm 14.00 \mathrm{~km}$ in the $x$ and $y$ axes from central location. Following after Ateya and Takemoto $(2002 a, b)$ the independent faces gravity anomaly effects were computed using Eqs. (2) and (3) respectively. The maximum gravity 


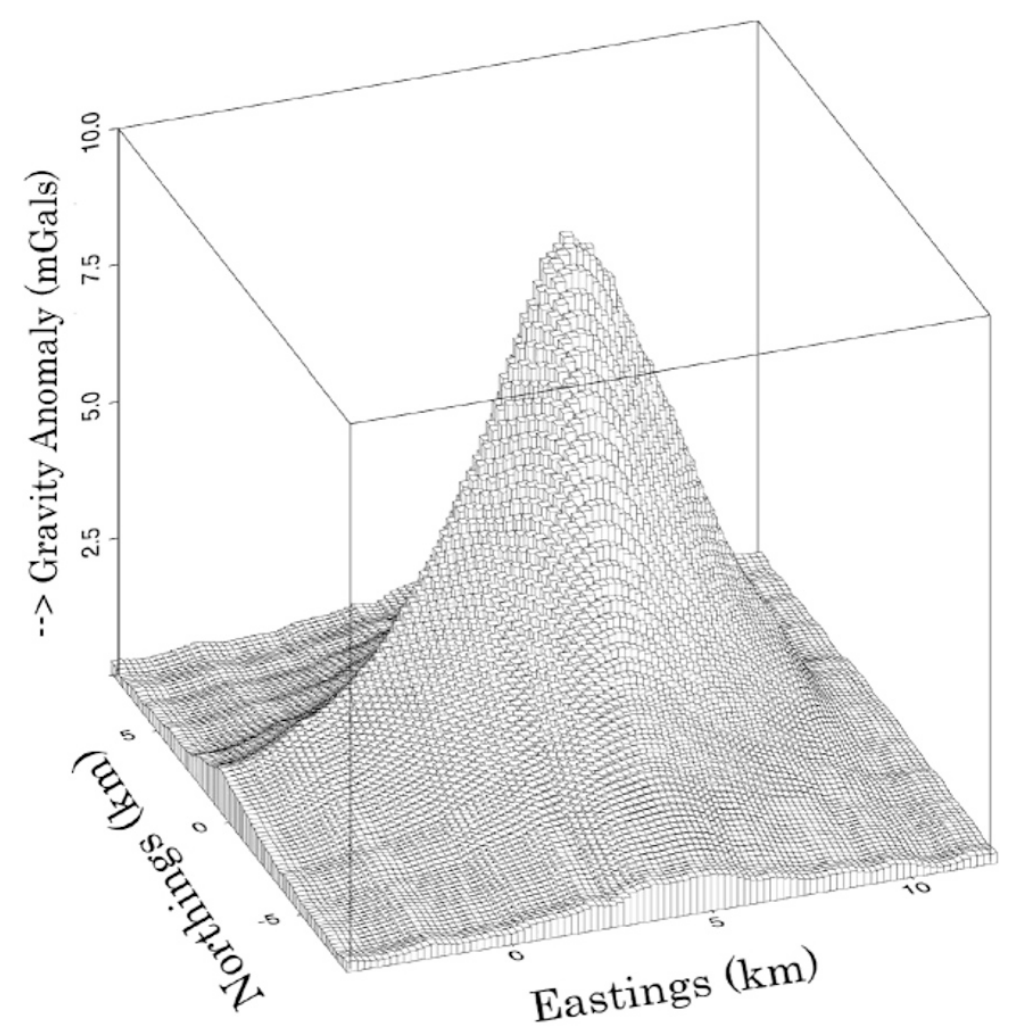

Fig. 4. Synthetic gravity anomaly effect of the three-dimensional sub-surface dipping dike displaced eastwards by $5.0 \mathrm{~km}$.

anomaly effect of the two faces was $9.50 \mathrm{mGals}$ as shown in Fig. 3.

The gravity anomaly effects of the two-dimensional independent faces were generated to cover the entire rectangular grid and superimposed onto each other with each co-ordinate having a gravity anomaly effect from each face interpolated directly above the grid nodes to form one overall system of gravity anomaly effects.

The resultant gravity anomaly effect was then further modeled and analyzed dependent on the co-ordinate locations to have a maximum anomaly equal to the maximum gravity anomaly effect of each independent face. The data was then contaminated by un-correlated Gaussian noise of maximum amplitude of $0.50 \mathrm{mGals}$. The final gravity anomaly effect was deliberately shifted by $5.00 \mathrm{~km}$ Eastwards to enable investigate for apparent shifts in the subsurface structure. The peak gravity anomaly effect was maintained at 9.50 mGals. The resultant three-dimensional synthetic gravity anomaly effect for the sub-surface dipping dike-like structure is shown in Fig. 4.

\section{Inversion Analysis}

Solving an inverse problem means making inferences about physical systems from observation data. The gravity inverse problem is an ill-posed problem in the sense of Hadamard (1902) because its solution is neither unique nor stable. The non-uniqueness of the inverse problem increases rapidly for bodies with in-homogenous density and rapidly becomes unmanageable. The stable solution is possible thanks to regularization techniques on the basis of the general principles enunciated by Tikhonov and Arsenin (1974) and/or Tikhonov and Glasko (1965).

The disturbing or gravitating masses in the lower halfspace $(z>0)$ can be reflected in the potential field observed on the Earth's surface. The problem of finding the different densities in the entire lower half-space below $h=H_{0}$ was previously considered in Savinsky (1984) where the limit for was at infinity instead of $H_{0}+\Delta H$. For similar cases the problem is to derive the greatest possible amount of information on the positions, locations and the sub-surface structures of the causative sources from the measurements of potential fields, e.g., the field observed gravitational anomalies, $\Delta g\left(x_{j}, y_{j}, z_{j}\right)$.

A possible sub-surface location in the horizontal layer can be at a depth from $h=H_{0}$ to $h=H_{0}+\Delta H$, where $\Delta H$ the thickness of the horizontal layer and $Q(x, y, h)$ the disturbing masses that represent the density distributions. If one assumes that there are values of the potential field $\Delta g\left(x_{j}, y_{j}, z_{j}\right), j=1,2 \ldots N$, where $z_{j}$ are deviations in the vertical direction from the horizontal measurements level $h=0$, then the solution of the problem can be achieved through Eq. (4):

$$
\begin{gathered}
G \int_{-\infty}^{+\infty} \int_{-\infty}^{+\infty} \int_{H_{0}}^{H_{0}+\Delta H} \frac{\left(z_{j}+h\right) Q(x, y, h) d x d y d h}{\left[\left(x-x_{j}\right)^{2}+\left(y-y_{j}\right)^{2}+\left(z_{j}+h\right)^{2}\right]^{3 / 2}} \\
=\Delta g\left(x_{j}, y_{j}, z_{j}\right) \quad j=1,2, \ldots, N
\end{gathered}
$$

where $G$ is the gravitational constant with the $x_{j}$ and $y_{j}$ as the $x$-axis and $y$-axis co-ordinates of the grid points (Savinsky et al., 1981). It is possible to obtain the density distributions $Q(x, y, h)$ within the horizontal layer in the polyno- 


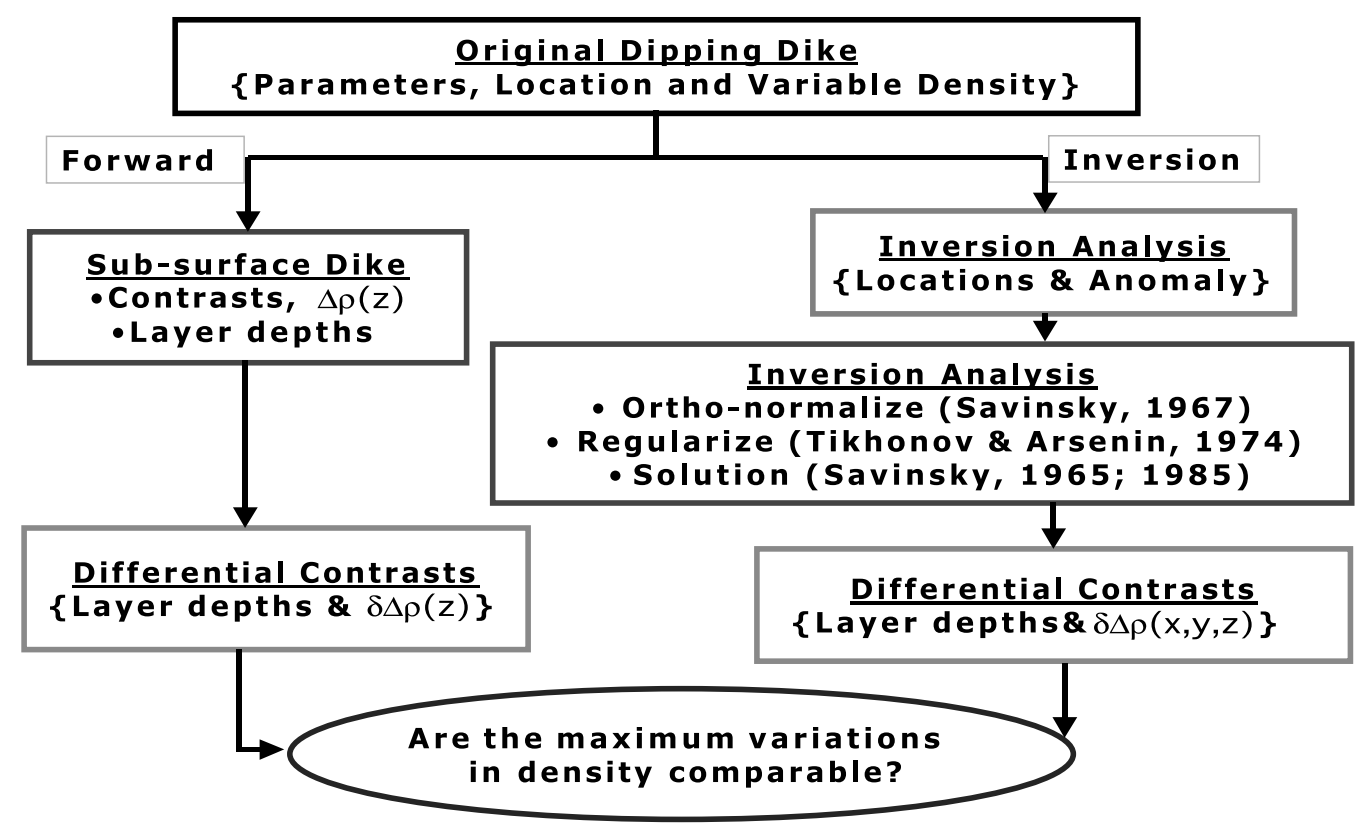

Fig. 5. Quantitative determination of the density contrasts in an intermediate horizontal layer.

Table 2. Differences between a priori and inversion analysis density contrasts.

\begin{tabular}{cccc}
\hline $\begin{array}{c}\text { Depth of layer } \\
(\mathrm{km})\end{array}$ & $\begin{array}{c}\text { Model density } \\
\text { contrasts }\left(\mathrm{g} / \mathrm{cm}^{3}\right)\end{array}$ & $\begin{array}{c}\text { Inversion analysis } \\
\text { contrasts }\left(\mathrm{g} / \mathrm{cm}^{3}\right)\end{array}$ & $\begin{array}{c}\text { Differences } \\
\left(\mathrm{g} / \mathrm{cm}^{3}\right)\end{array}$ \\
\hline 0.000 & 0.000 & 0.000 & 0.000 \\
0.400 & 0.032 & 0.028 & 0.004 \\
0.800 & 0.042 & 0.045 & -0.003 \\
1.200 & 0.042 & 0.038 & 0.004 \\
1.600 & 0.041 & 0.046 & -0.005 \\
2.000 & 0.039 & 0.054 & -0.015 \\
\hline
\end{tabular}

Table 3. Location of investigation site in Chubu District.

\begin{tabular}{lcccc}
\hline & $\begin{array}{c}\text { Longitude } \\
\text { (Deg.) }\end{array}$ & $\begin{array}{c}\text { Latitude } \\
\text { (Deg.) }\end{array}$ & $\begin{array}{c}\text { Height above } \\
\text { sea level }(\mathrm{m})\end{array}$ & $\begin{array}{c}\text { Residual anomaly } \\
\text { (mGals) }\end{array}$ \\
\hline Minimum & 137.875 & 36.320 & 500.342 & -5.425 \\
Maximum & 137.975 & 36.420 & 702.808 & 8.175 \\
Difference & 0.100 & 0.100 & 202.466 & 13.600 \\
\hline
\end{tabular}

mial form, the terms of which are formed by the functions of the kernel of the integral equation (Savinsky, 1963; Savinsky et al., 1981). The resulting density distribution or disturbing masses $Q(x, y, h)$ in Eq. (4) have a physical meaning only at $H_{0}<h<H_{0}+\Delta H$ and therefore a series of horizontal layers taken to cover the entire lower half-space.

Figure 5 shows the flow diagram that incorporates subsurface model; the procedure of the inversion analysis for the determination of the variable density contrasts in a series of intermediate horizontal layers. The differences between the differential density contrasts from forward modeling and inversion analysis for each horizontal layer are given in Table 2 .

Table 2 shows the inversion analysis contrasts where the heights of the horizontal layers are kept constant at $\Delta H=$ $0.400 \mathrm{~km}$ although several different horizontal layer heights were actually investigated. The second column represents the differential density contrasts from the forward model $\Delta \rho(z)$ using a reduction density of $2.645 \mathrm{~g} / \mathrm{cm}^{3}$ given that $\Delta \rho(0)=-0.525$ while the third column represents the maximal density contrasts $\Delta \rho(x, y, z)$ in each respective horizontal layer. Fourth column shows the differences between the a priori and recovery density contrasts. Figure 6 shows density contrasts for an intermediate horizontal layer of depth range $0.400 \sim 0.800 \mathrm{~km}$ with a contour interval of $0.003 \mathrm{~g} / \mathrm{cm}^{3}$.

\section{Application with Actual Field Data}

An investigation site in Matsumoto basin, Chubu District was chosen for the inversion analysis with the actual field location as given in Table 3. It covers an area of approximately $9.0 \mathrm{~km}$ by $9.0 \mathrm{~km}$ with an almost flat terrain. The 


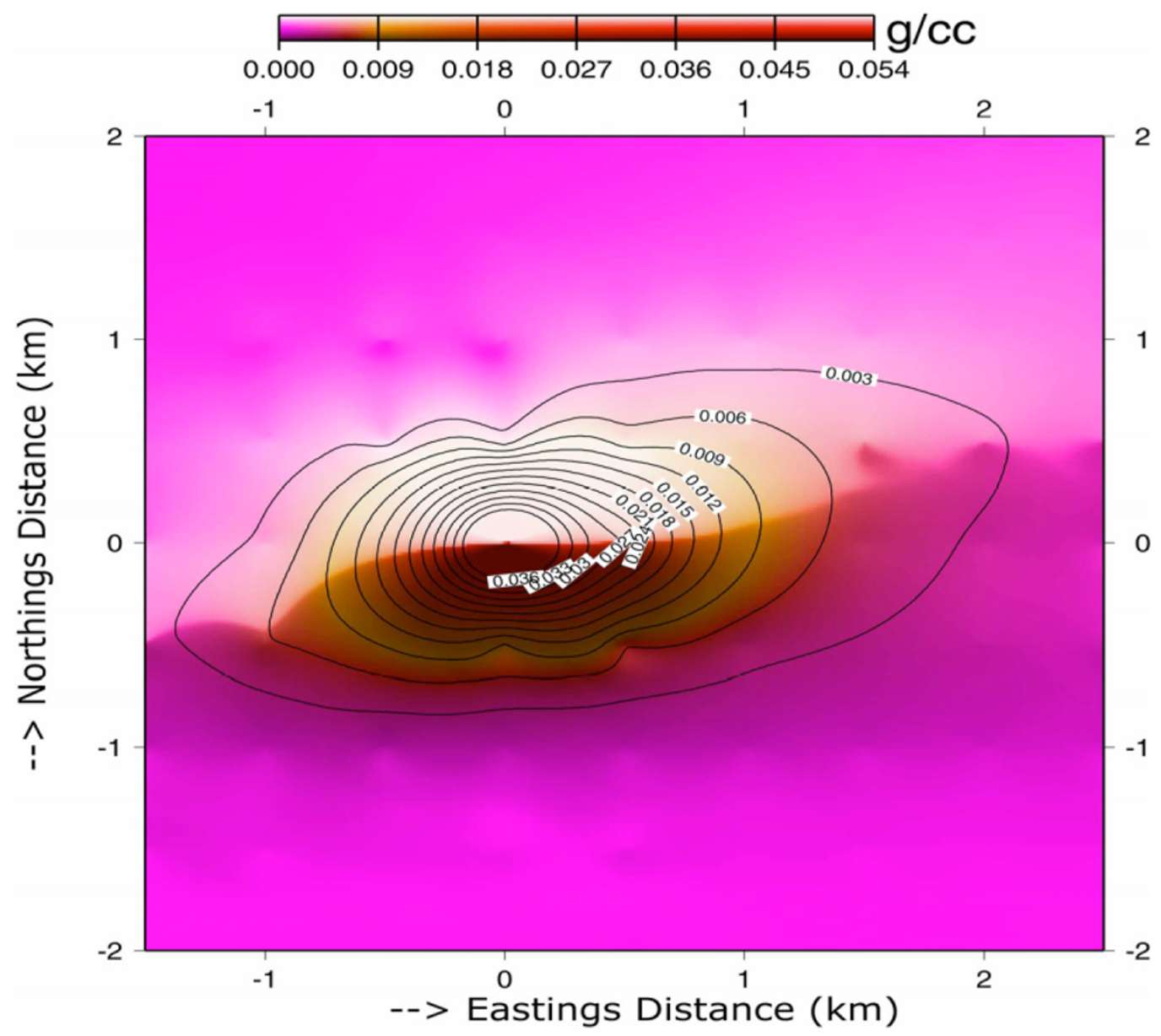

Fig. 6. Density contrasts for an intermediate horizontal layer of depth range $0.400 \sim 0.800 \mathrm{~km}$ with a contour interval of $0.003 \mathrm{~g} / \mathrm{cm}^{3}$.
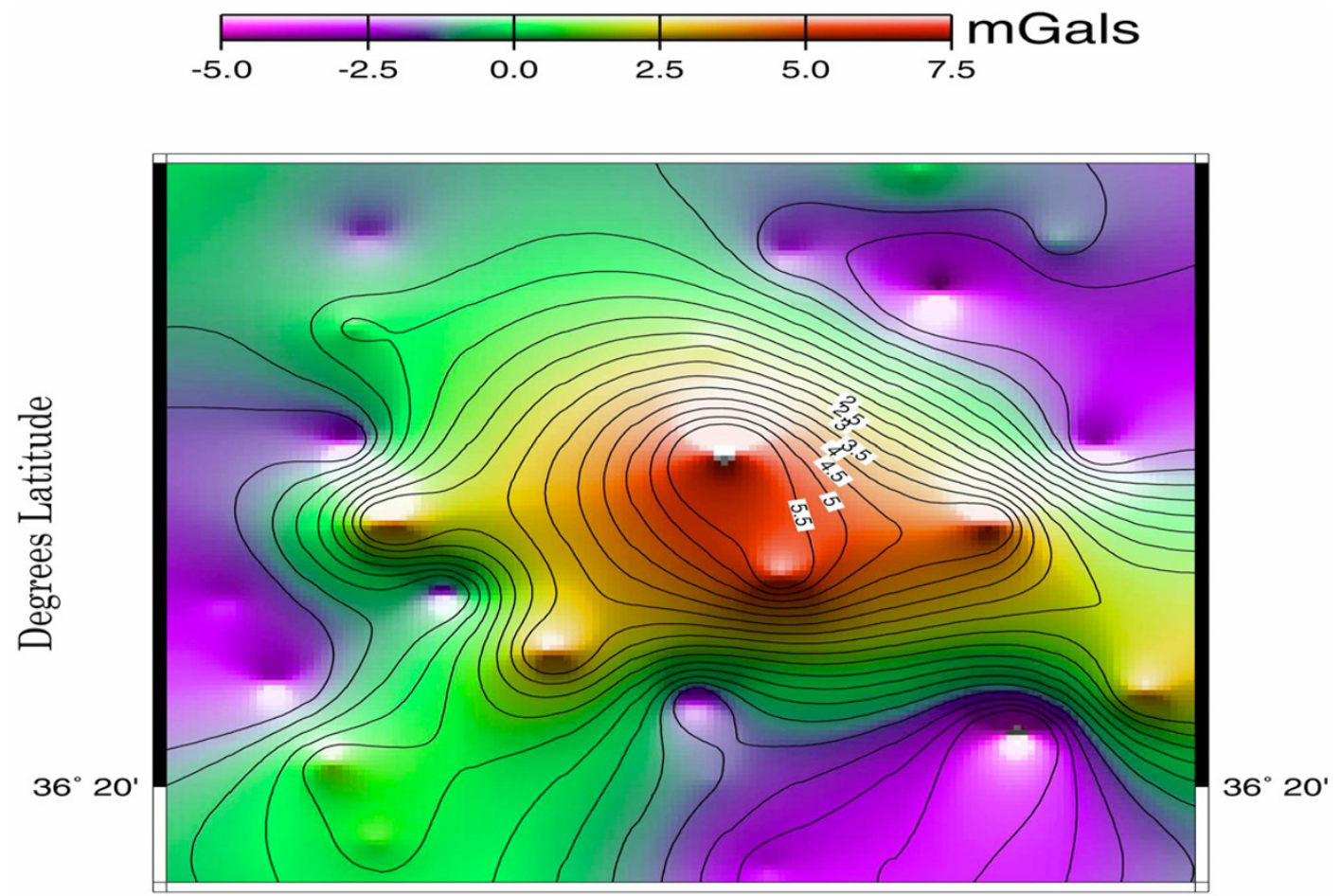

Degrees Longitude

Fig. 7. Residual anomaly of the investigation site in Matsumoto Basin, Chubu District—Japan. 
Table 4. Density contrasts for layers in investigation site.

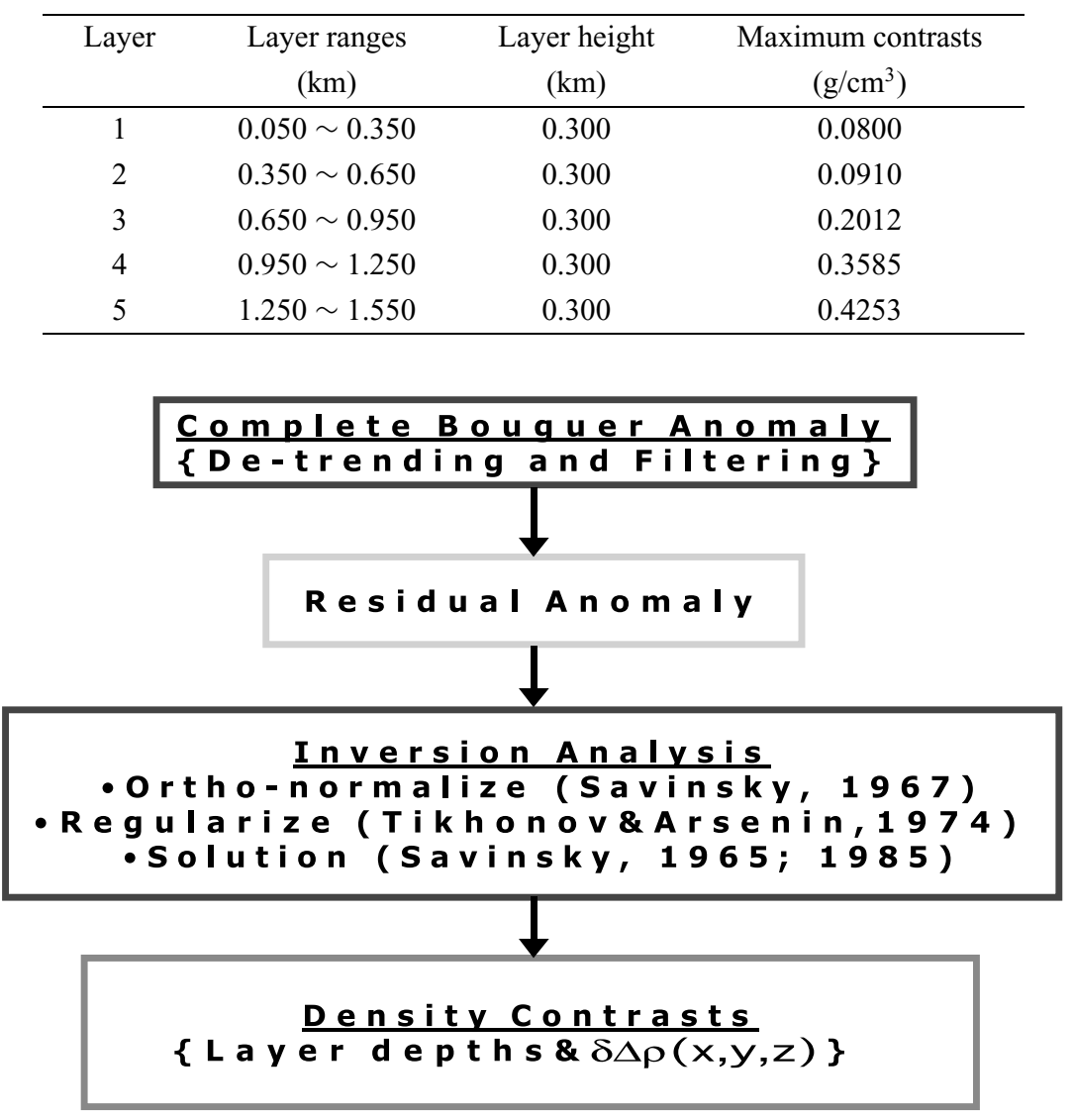

Fig. 8. Determination of the density contrasts in a horizontal layer using residual anomaly.

complete Bouguer anomaly of the region was computed with the adopted average topographic density as $2.64 \mathrm{~g} / \mathrm{cm}^{3}$ after of Yamamoto et al. (1982).

A major step in the analysis of the gravity data is the process of isolating the observed anomaly patterns into regional and residual components (Chapin, 1996). The complete Bouguer anomaly of the encompassing region was computed and the residual anomaly determined for the investigation site given in Table 3 using data obtained from Geological Survey of Japan (2000). Figure 7 gives the residual anomaly of the investigation site that was utilized.

In both de-trending and filtering we utilized the GMT software prepared by Wessel and Smith (1995). The computation of the density contrasts in a series of horizontal layers with different heights in the sub-surface proceeds as in Fig. 8. It helps determine the depth-dependent density contrasts $\Delta \rho(x, y, z)$ from the residual anomaly by inversion analysis. The point-to-point separation was maintained at $0.50 \mathrm{~km}$ in the final interpolated values on the grid. The inversion analysis results for the density contrasts for a subsurface horizontal layer of range $0.350 \sim 0.650 \mathrm{~km}$ are shown in Fig. 9.

Similar inversion analysis results for a series of horizontal layers of same thickness, i.e., each with a layer height of $0.300 \mathrm{~km}$ was performed and the summary is given in Table 4 . The inversion analysis was performed to a depth of $1.60 \mathrm{~km}$. The contour of the disturbing masses gives a possible density contrasts in the structure at the location.
Table 4 shows the maximum density contrasts for a possible density contrasts structure in Matsumoto basin, Chubu District. The density or density contrasts of the sub-surface could then be obtained by relating the density or density contrasts to the Earth surface between the subsequent intermediate horizontal layers.

\section{Discussion and Conclusions}

It has been shown that it is possible to determine or recover the intermediate layer density contrasts $\Delta \rho(x, y, h)$ changes with respect to depth from inversion analysis. The error differences are less than $5.0 \mathrm{mg} / \mathrm{cm}^{3}$ up to a depth of 2.00 $\mathrm{km}$ for the horizontal layers of $0.400 \mathrm{~km}$ thickness have been realized.

In this case the depth-dependent density contrasts forward model $\Delta \rho(z)$ was computed a priori for a series of intermediate horizontal layers with varying heights and at different depths. The same heights were utilized in the inversion analysis to determine if it is possible to recover the maximum values i.e., quantities in the forward model.

The differences increase gradually after a depth of 2.00 $\mathrm{km}$ in the sub-surface. One possibility for the increase is due to use of greater point-to-point separation distance with increasing depth. It effectively smoothens the effective gravity anomaly effect as noted in Savinsky (1967), i.e., set of inversion results become poorly defined at increasing depths $H_{0}$ due the influence of the accumulated errors or smoothing of the gravity anomaly effect with increase in the point- 


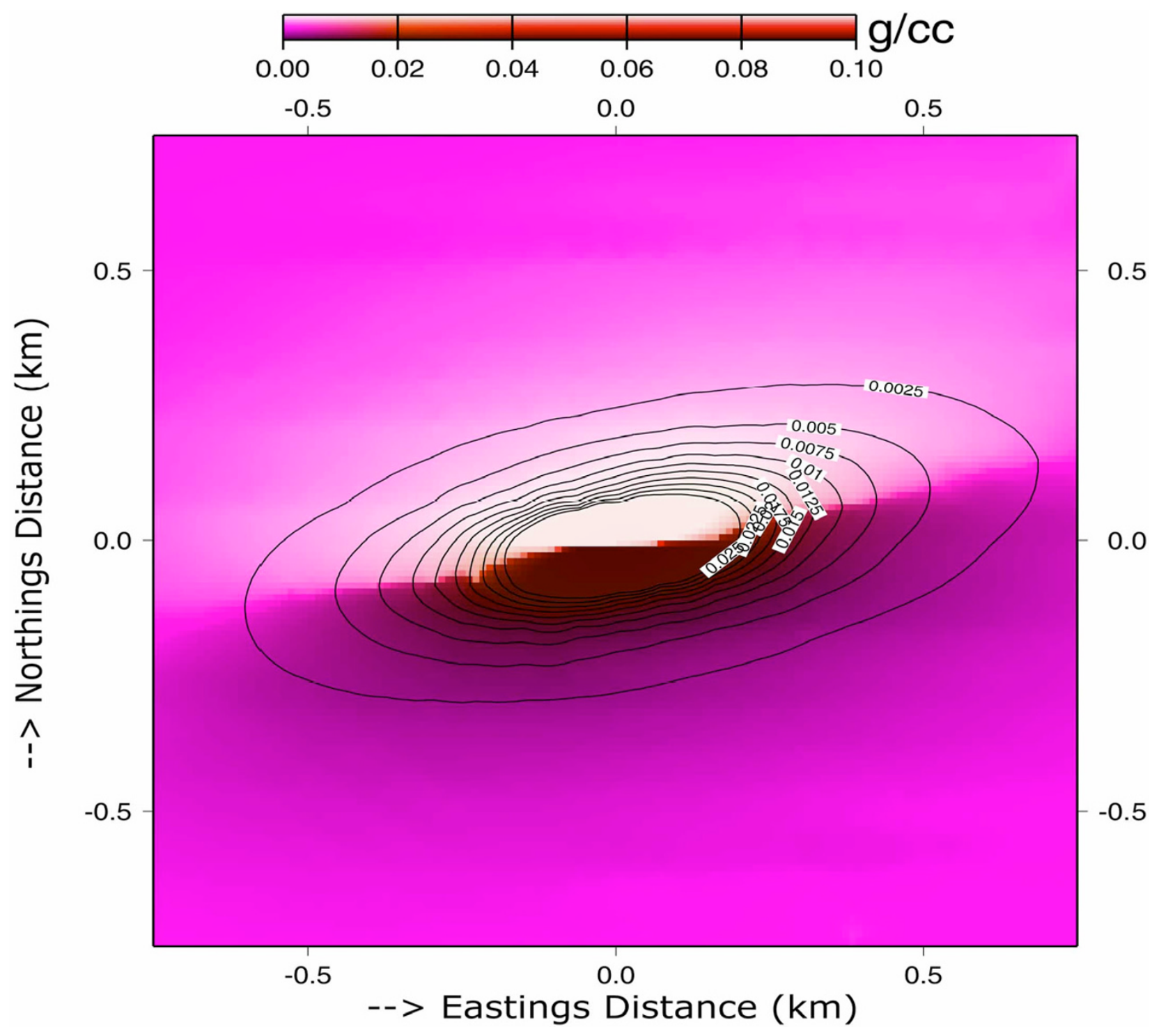

Fig. 9. Density contrasts results for a horizontal layer of range $0.350 \sim 0.650 \mathrm{~km}$ with a contour interval of $0.0025 \mathrm{~g} / \mathrm{cm}^{3}$.

to-point separations. These accumulated errors calls for the need for regularization techniques applied in the inversion analysis (Tikhonov and Arsenin, 1974; Koch, 1990).

We chose the maximum values (quantities) per intermediate layer because of the maximum gravity anomaly effect maintained at either central or shifted locations. Each maximum value mirrors the highest value of density contrasts for each intermediate layer possible from the synthetic dipping dike model. Similar inversion analysis was applied to an investigation site for a real field data in Matsumoto basin, Chubu District.

Results of the investigation site for a possible sub-surface structure in Matsumoto basin, Chubu District are depicted in fourth column of Table 4 with maximum density contrasts for a series of intermediate horizontal layers. In conclusion in the present study, the determination of a priori depthdependent density contrasts within an intermediate horizontal layer has been quantitatively demonstrated. Closely related to the disturbing masses in an intermediate horizontal layer the recovery of density contrasts in the actual location of the causative structure in the sub-surface has been demon- strated too. The determination of disturbing masses is more effective in micro-gravimetry studies and/or localized structures without the effects of long-wavelength potential field anomalies and a more accurate interpretation is dependent on the abundance of geological information.

Acknowledgments. We would like to express our gratitude to Geological Society of Japan (GSJ) for the entire gravity data and part of the geological data utilized in this research as provided on the Gravity CD-ROM for Japan dated 24th March 2000. Further our thanks to Geographical Survey Institute (GSI) for the Digital Terrain Data for the Japan Alps region and the extra gravity data especially in Matsumoto Basin.

\section{References}

Ateya, I. L. and S. Takemoto, Inversion of gravity across a sub-surface dike-like structure in two dimensions. Western Pacific Geophysics Meeting (WPGM), Wellington, New Zealand, EOS Trans. AGU, 83(22), 112, 2002a.

Ateya, I. L. and S. Takemoto, Gravity inversion modeling across a 2-D dikelike structure-A Case Study, Earth Planets Space, 54, 791-796, 2002 b. Boulanger, O. and M. Chouteau, Constraints in 3-D gravity inversion, Geophysical Prospecting, 49, 265-280, 2001.

Chapin, D. A., A deterministic approach toward isostatic gravity residuals- 
A case study from South America, Geophysics, 61(4), 1022-1033, 1996. Cordell, L., Gravity analysis using an exponential density-depth functionSan Jacinto Graben, California, Geophysics, 38, 684-690, 1973.

Geldart, L. P., E. D. Gill, and B. Sharma, Gravity anomalies of twodimensional faults, Geophysics, 31(2), 372-397, 1966.

Geological Survey of Japan (GSJ), Gravity CD-ROM of Japan published on 24th March 2000, URL http: / / www.gsj .go.jp.

Guspi, F., General 2-D gravity inversion with density contrasts varying with depth, Geo-exploration, 26, 253-265, 1990.

Hadamard, J., Sur les problemes aux derive espartielles et leur signification physique, Bulletin of Princeton University, 13, 1-20, 1902.

Koch, M., Optimal regularization of the linear seismic inverse problem, Technical Report No. FSU-SCRI-90C-32, Florida State University, Tallahassee, Florida, 1990.

Li, Y. and D. W. Oldenburg, 3-D inversion of magnetic data, Geophysics, 61, 394-408, 1996.

Li, Y. and D. W. Oldenburg, 3-D inversion of gravity data, Geophysics, 63(1), 109-119, 1998.

Martin-Atienza, B. and J. Garcia-Abdeslem, 2-D gravity modeling with analytically defined geometry and quadratic polynomial density functions, Geophysics, 64, 1730-1734, 1988.

Murthy, I. V. R. and D. B. Rao, Gravity anomalies of two-dimensional bodies of irregular cross-section with density contrast varying with depth, Geophysics, 44, 1525-1530, 1979.

Rao, D. B., Analysis of gravity anomalies over an inclined fault with quadratic density function, Pageoph, 123, 250-260, 1986a.

Rao, D. B., Modeling of sedimentary basins from gravity anomalies with variable density contrast, Geophysics Journal of the Royal Australian Society, 84, 207-212, 1986b.

Rao, D. B., Analysis of gravity anomalies of sedimentary basins by asymmetrical trapezoidal model with quadratic function, Geophysics, 55, 226$231,1990$.

Ruotoistenmaki, T., The gravity anomaly of two-dimensional sources with continuous density distribution and bounded by continuous surfaces, Geophysics, 57(4), 623-628, 1992.

Savinsky, I. D., On solving inverse geophysical problems represented by the Fredholm integral equations of the first order, Izvestiya Academy USSR Ser. Geofiz, No. 5, 712-721, 1963.

Savinsky, I. D., On solving incorrect problems of recalculation of the potential field to underlying levels, Izvestiya Academy of Sciences, USSR Physics of Solid Earth, No. 6, 72-92, 1967.

Savinsky, I. D., Solving inverse problems on gravity data with help of kern functions of integral equations, in Applied Geophysics, 109, pp. 86-95, Nedra, Moscow, 1984.

Savinsky, I. D., On determination of the contact interface from gravitational and magnetic field, Physics of the Solid Earth, English Translation, 30, No. 10, pp. 903-907, 1995.

Savinsky, I. D., V. L. Briskin, and A. A. Petrova, Reduction of the Gravitational and Magnetic inclined and Vertical Planes in the Lower HalfSpace, Izvestiya Earth Physics, 17(12), 934-943, 1981.

Telford, W. M., L. P. Geldart, and R. E. Sheriff, Applied Geophysics, 2nd Edition, Cambridge University Press, New York, 1990.

Tikhonov, A. N. and A. Y. Arsenin, Methods of Solving Incorrectly Posed Problems, Nauka, 224, Moscow, 1974

Tikhonov, A. N. and V. B. Glasko, Application of the regularization method in nonlinear problems, J. Comp. Math. Math. Phys, 5(3), 363-373, 1965.

Wessel, P and W. H. F. Smith, The Generic Mapping Tools (GMT) Version 3.0 Technical Reference and Cookbook, SOEST/NOAA, 1995.

Yamamoto, A., K. Nozaki, Y. Fukao, M. Furumoto, R. Shichi, and T. Ezaka, Gravity survey in the Central Ranges, Honshu, Japan, Journal of Physics of the Earth, 30, 201-243, 1982.

Zhang, J., B. Zhong, X. Zhou, and Y. Dai, Gravity anomalies of 2-D bodies with variable density contrast, Geophysics, 66(3), 809-813, 2001.

I. L. Ateya (e-mail: ateya@kugi.kyoto.ac.jp) and S. Takemoto 